• 研究报告・

\title{
基底颜色对两种沙蜥体色变异的影响
}

\author{
童浩杰 ${ }^{1}$ 张凯龙 ${ }^{1}$ 刘宇航 ${ }^{1}$ 张立勋 ${ }^{2}$ 赵 伟 ${ }^{2}$ 金园庭 $1^{*}$ \\ 1 (中国计量大学生命科学学院, 杭州 310018) \\ 2 (兰州大学生命科学学院, 兰州 730000)
}

\begin{abstract}
摘要: 生存在不同基底颜色环境下的爬行动物种群通常表现出丰富的体色地理变异, 其体色变化的潜在机制具有 多样性。变色沙蚘(Phrynocephalus versicolor)和草原沙蚚( P. frontalis)具有较近的遗传关系, 曾被认为与荒漠沙蚚 (P. przewalskii)组成同一系统发育种组。本文应用光纤光谱仪(AvaSpec-2048), 通过记录沙蚚背部体表12个部位的 皮肤光反射率, 定量比较在黑化环境下的深色变色沙蚚与非黑化环境下的浅色草原沙蚚自然体色变异, 研究其种 群体色变异是否具有时间可逆性, 并探讨基底颜色对沙蚚体色的影响机制。研究结果表明, 黑化生境下的变色沙 蝰体色显著深于非黑化枯黄色生境下的草原沙衫。此外, 对黑化与非黑化样本开展的生境互换移植围栏实验, 即 把枯黄色生境中非黑化的草原沙蚚移植于黑色的基底环境中饲养, 把黑化生境中黑化的变色沙蜥移植于枯黄色生 境中饲养。结果表明, 饲养1周后黑化群体背部6个检测部位的光反射率显著变大, 其他部位均无显著变化; 而非黑 化群体只有左后肢和背部右上方 2 个部位的皮肤光反射率发生显著变化, 其他部位反射率无显著变化。结果表明, 变色沙蚚体色变异能力比草原沙蝰强, 体色表型可能已经在两个近缘沙蚚物种中稳定遗传, 基底生境颜色的短期 变化在统计学上能引起肉眼难以识别的轻微的体色变异, 个体发育相关的一些遗传因素可能对体色变异起控制 作用。
\end{abstract}

关键词: 黑化; 体色变异; 沙蜥; 皮肤反射率

\section{Effects of substrate color on the body color variation of two agamid liz- ards, Phrynocephalus versicolor and $P$. frontalis}

\author{
Haojie Tong ${ }^{1}$, Kailong Zhang ${ }^{1}$, Yuhang Liu ${ }^{1}$, Lixun Zhang ${ }^{2}$, Wei Zhao ${ }^{2}$, Yuanting Jin ${ }^{1 *}$ \\ 1 College of Life Sciences, China Jiliang University, Hangzhou 310018 \\ 2 College of Life Sciences, Lanzhou University, Lanzhou 730000
}

\begin{abstract}
Geographical variation of body color is widely present in reptile populations that survive in different substrate habitats, multiple potential mechanisms can account for this color variation. Phrynocephalus versicolor and $P$. frontalis, close genetic relatives, constitute a phylogenetic species group together with $P$. przewalskii. In this study, a fiber spectrophotometer (AvaSpec-2048) was used to record the skin luminous reflectivity of 12 sites across the lizard's body, and we quantitatively compared the natural color variation of dark $P$. versicolor and light $P$. frontalis that lived in "melanistic" and "non-melanistic" habitats, respectively. We aimed to determine whether the color variations of both populations were time reversible, and further discuss potential mechanisms that substrate color may have on color variation of Phrynocephalus lizards. Our results showed that the body color of $P$. versicolor in "melanistic" habitat was significantly darker than $P$. frontalis in the "non-melanistic" withered yellow habitat. We also conducted a reciprocal transplantation experiments (i.e. "non-melanistic" withered yellow $P$. frontalis individuals were transplanted and fed in "melanistic" substrate environment, while "melanistic" P. versicolor individuals were transplanted and fed in withered yellow substrate environment). For "melanistic" P. versicolor, the skin reflectivity of six sites increased significantly after one week, while no significant changes were detected in other sites. For "non-melanistic" P. frontalis, except the skin reflectivity of two sites (left hind limb and top right on the
\end{abstract}

收稿日期: 2016-04-29; 接受日期: 2016-07-08

基金项目: 国家自然科学基金(31372183)

* 通讯作者 Author for correspondence. E-mail: jinyuanting@126.com 
back) significantly changed, compared to corresponding values one week previously, other sites showed no significant changes. Our results suggest that $P$. versicolor possesses stronger color variation ability than $P$. frontalis, and the color phenotypes are likely inherited in both species. Short-term changes of substrate color can cause slightly color variations that are difficult to distinguish by naked eyes, suggesting ontogeny related hereditary factors may also play a controlling role.

Key words: melanistic; body color variation; Phrynocephalus; skin reflectivity

受到自然选择作用, 动物能表现出丰富的体色 变异, 在其避敌、交流等方面发挥重要作用(Barlett \& Gates, 1966; Geen \& Johnston, 2014)。一些动物的 体色会随着所处基底颜色而变化, 如头足动物 (Hanlon, 2007)、甲壳动物(Hemmi et al, 2006)、鱼 (Moretz \& Morris, 2003)、两栖动物(Stegen et al, 2004), 以及乌龟(Woolley, 1957)、变色树蚚(Calotes versicolor)(蔡波, 2014)、变色龙(Bradypodion taeniabronchum)(Stuart-Fox et al, 2008)等一些爬行动 物。此类体色变化可能与表型可塑性或者遗传因素 相关(Vroonen et al, 2012)。

爬行动物的体色变异有两种主要形式: 一种是 生理体色变化, 它是对短期环境刺激的适应, 一般 通过色素扩散与聚集而快速地实现与基底颜色的 匹配(Nery \& Castrucci, 1997); 另一种是形态体色 变化, 这种变化是对长期环境刺激的适应, 通常与 色素的产量有关(Rosenblum, 2004)。一些爬行动物 的生理体色变化可以在几毫秒或者几小时内实现 (Thurman, 1988), 而形态体色变化可能需要数天或 者数月, 并通常受众多遗传因素的影响(蔡波, 2014)。

沙蝰属(Phrynocephalus)属于有鳞目、爬行纲、 镾蜥科, 约有 40 个物种(赵尔密等, 1998, 1999)。沙蚚 广泛分布于不同自然基底色的开阔生境中, 其种群 体色表现出丰富的地理变异。变色沙蚚 ( P. versicolor)和草原沙蚚 (P. frontalis)均属于卵生物种, 两 者广泛分布于中国北部和西北部, 包括新疆、内蒙 古、甘肃以及宁夏的荒漠和半荒漠地区(赵尔密等, 1999)。基于线粒体基因的分子系统学研究认为, 分 布于新疆及其东部较高地区的沙蚚(变色沙蚚、草原 沙蚚与荒漠沙蜊)起源于共同祖先, 被视为一个种 组(Wang \& Fu, 2004)。目前, 对该种组的生态学研 究主要还是集中于食性(全仁哲等, 2006; 赵雪等, 2013), 繁殖策略(陈强等, 1993; 郭砺和赵辰光, 2001)、两性异形(Qu et al, 2011)等方面, 未见有关其
体色变异的研究报道。我们在甘肃瓜州县的柳园镇 黑山口附近发现的变色沙蚚躯体明显黑化, 而与分 布在枯黄色生境中的与其近缘的草原沙蚚具有显 著的体色差异。由此可见, 不同基底色环境下生存 的变色沙蜊种组的不同类群是研究动物体色变异 机制的良好模型。

近年来, 光谱技术开始逐渐应用于动物体色研 究(杨灿朝等, 2011; 杨灿朝和梁伟, 2013), 它能对 动物体表颜色变化进行量化分析, 包括对紫外光、 可见光以及红外光区的探测, 为生物体体色生态学 研究提供了强大的技术支持。不同颜色的皮肤对光 的反射率存在差异, 黑化的皮肤相对于浅色的皮肤 具有更低的光反射率(Porter \& Gates, 1969)。本文应 用光谱技术, 比较了在黑化与非黑化生境下生存的 两个沙蚚近缘种(分别为变色沙蜥和草原沙蚚)的体 色差异，旨在评估基底颜色对沙蚚体色变异的影 响, 并采用生境互换移植实验研究两种沙蚚体色变 异是否具有可逆性，区分其体色变异的具体类型。

\section{1 材料与方法}

\section{1 采样、饲养与围栏实验}

30条黑化的变色沙蚚成体 $(15 \hat{\circ}, 15$ ㅇ)采集自甘 肃省瓜州县柳园镇的黑山口 $\left(95.47^{\circ} \mathrm{E}, 41.01^{\circ} \mathrm{N}\right)$, 海拔1,683 m; 30条非黑化的草原沙虲成体(15万, 15 ㅇ)采集自内蒙古自治区巴彦淖尔市乌拉特前旗 $\left(108.73^{\circ} \mathrm{E}, 40.75^{\circ} \mathrm{N}\right)$, 海拔1,080 m (图1)。沙蚚样本 于2015年8月采集后剪趾标记，分 A、B两类(A基底 使用黑沙石, $\mathrm{B}$ 使用枯黄色沙石)饲养于兰州大学实 验室。采用 $0.60 \mathrm{~m} \times 0.45 \mathrm{~m} \times 0.35 \mathrm{~m}$ (长 $\times$ 宽 $\times$ 高) 的浅 白色塑料盒围栏养殖, 每个盒子下方铺上 $3-5 \mathrm{~cm}$ 厚 的沙石, 沙石采集自样本自然种群生境。在白天, 盒子内的部分区域可以受到阳光照射以供蚚蜴调 节体温。此外, 给蜥蜴提供充足的食物(黄粉虫幼虫) 和饮水。将 30 条非黑化的草原沙蚚饲养于 2 个 $\mathrm{A}$ 盒, 30 条黑化的变色沙蚚饲养于 2 个 $\mathrm{B}$ 盒。部分样本移植 
A

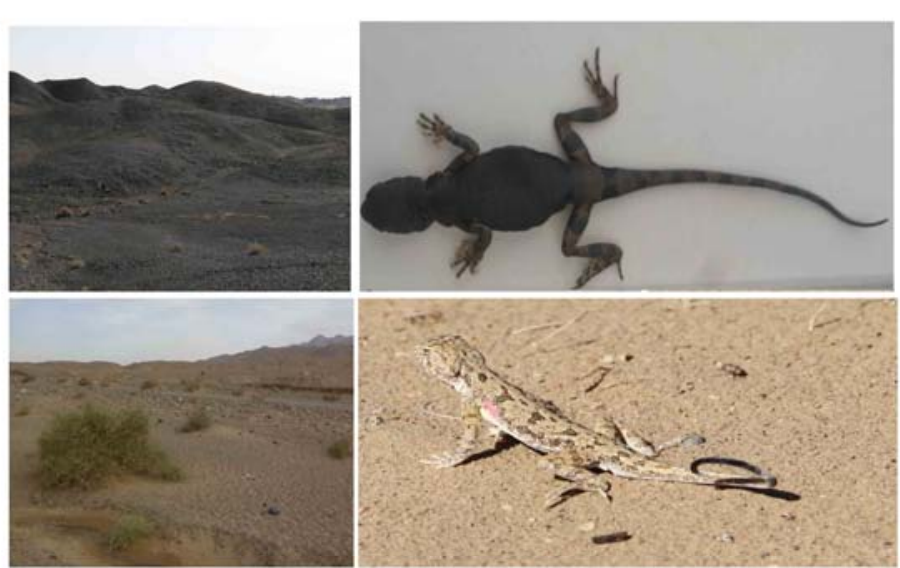

B

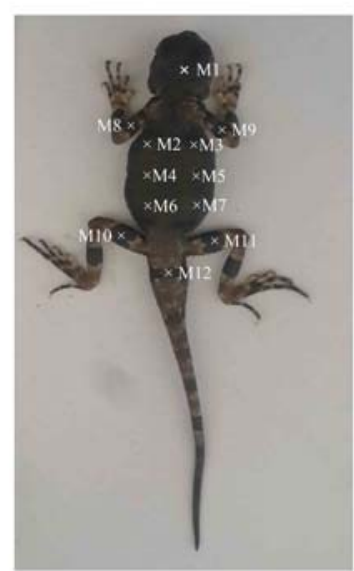

图1 沙蚚体色及体色测定点选择。(A)黑化的变色沙蚚采集自黑色基底的生境(上), 非黑化的草原沙蚘采集自枯黄色基底的 生境(下); (B) M1至M12分别代表蜊蜴个体体色测量的12个部位, 包括头顶部中心(M1)、背部左上方(M2)、背部右上方(M3)、 背中部左侧(M4)、背中部右侧(M5)、背部左下方(M6)、背部右下方(M7)、左前肢(M8)、右前肢(M9)、左后肢(M10)、右后 肢(M11)和尾根部背侧(M12)。

Fig. 1 Body color of lizards and the selected sites for color measurement. (A) "Melanistic" P. versicolor were collected from black substrate habitat (above), "non-melanistic" P. frontalis were sampled from withered yellow substrate habitat (below); (B) M1-12 represent twelve color measuring sites for each lizard, including cranial center (M1), top left on the back (M2), top right on the back (M3), left side on the central back (M4), right side on the central back (M5), bottom left on the back (M6), bottom right on the back (M7), left forelimb (M8), right forelimb (M9), left hind limb (M10), right hind limb (M11), and tail root (M12).

实验后经处理用于后续分子实验。

\section{2 体色测量}

个体体色的测量涉及12个部位(图1)。采用荷兰 Avantes公司生产的AvaSpec-2048光纤光谱仪进行 测量, 该仪器由光谱仪主机AvaSpec-2048-USB2、光 源AvaLight-DH-S、反射探头FCR-7UV400-2-ME、 探头固定器RPH-1和白色漫射板WS-2等部件组成。 光谱采样间隔为 $0.60 \mathrm{~nm}$, 波段范围为 $200-1,100 \mathrm{~nm}$ (杨灿朝等, 2011), 涵盖了有鳞目及其捕食者鸟类 300-700 nm的视觉范围(Bennett \& Cuthill, 1994)。用 探头固定器固定反射探头, 预热主机后用白色漫射 板WS-2校准, 在垂直蚚蜴检测皮肤部位表面和距 离约 $2 \mathrm{~mm}$ 的位置测量发射光谱, 曝光时间设为 100 $\mathrm{ms}$ 。每个蚚蜴个体抓捕 2 天后测量 1 次, 生境互换移 植饲养后第8、9、10天分别测量1次, 共测量4次。 每次对所有个体的体色测量均在上午9-11点进行。

\section{3 数据分析}

光谱数据用配套软件AvaSoft 8.1提取和转换, 提取光谱范围为300-700 $\mathrm{nm}$ 的光反射率值并用于 后续分析。Origin8.0用于图形制作, SPSS20.0软件用 于统计学分析。用配对 $t$ 检验比较分析各部位初次测 定的光反射率分别与第8、9、10天的光反射率之间 的差异。描述性统计值用平均值土标准误表示, 显著
性水平设置为 $P<0.05$ 。

\section{2 结果}

分析结果显示, 黑化的变色沙蚚与非黑化的草 原沙蚚种群个体各部位光反射率均存在显著差异 (M1: $t_{29}=8.273, P<0.001 ;$ M2: $t_{28}=9.239, P<$ 0.001; M3: $t_{29}=9.533, P<0.001 ;$ M4: $t_{28}=10.089, P$ $<0.001$; M5: $t_{29}=10.572, P<0.001 ;$ M6: $t_{26}=$ 11.959, $P<0.001$; M7: $t_{29}=10.923, P<0.001$; M8: $t_{18}=5.192, P<0.001$; M9: $t_{20}=6.579, P<0.001$; M10: $t_{19}=6.959, P<0.001 ;$ M11: $t_{19}=7.258, P<$ 0.001; M12: $t_{18}=9.010, P<0.001$ )。

经过基底环境互换饲养后发现沙蚚样本体色 变化很轻微, 实际上肉眼难以识别判定, 更无法定 量。黑化与非黑化沙蜊种群各部位4次光谱测定的 反射率统计描述值见表 1 。配对 $t$ 检验结果(表2)显示, 在黑化的变色沙蚚种群中, 背部区域(M2-M7)第1 天的皮肤光反射率与环境移植饲养后第 8 天、第 9 天 及第 10 天的皮肤光反射率均存在显著差异(M5第 10 天除外), 而其他部位移植饲养前后的皮肤光反射 率并无显著差异; 非黑化的草原沙蜊种群中, 背部 右上方(M3)区域第1天的皮肤光反射率与移植饲养 后第 8 天及第 9 天的皮肤光反射率存在显著差 
表1 黑化的变色沙蜥与非黑化的草原沙蚚各检测部位的反射率(平均值土标准误)

Table 1 Mean values of reflectivity of each site for both "melanistic" P. versicolor and "non-melanistic" P. frontalis

\begin{tabular}{|c|c|c|c|c|c|c|c|c|}
\hline \multirow{2}{*}{$\begin{array}{l}\text { 检测部位 } \\
\text { Sites }\end{array}$} & \multicolumn{4}{|c|}{ 非黑化草原沙蜊 “Non-melanistic” P. frontalis } & \multicolumn{4}{|c|}{ 黑化变色沙蜥 “Melanistic” P. versicolor } \\
\hline & $\begin{array}{l}\text { 第1天 } \\
\text { 1st day }\end{array}$ & $\begin{array}{l}\text { 第8天 } \\
\text { 8th day }\end{array}$ & $\begin{array}{l}\text { 第9天 } \\
\text { 9th day }\end{array}$ & $\begin{array}{l}\text { 第10天 } \\
\text { 10th day }\end{array}$ & $\begin{array}{l}\text { 第1天 } \\
\text { 1st day }\end{array}$ & $\begin{array}{l}\text { 第8天 } \\
\text { 8th day }\end{array}$ & $\begin{array}{l}\text { 第9天 } \\
\text { 9th day }\end{array}$ & $\begin{array}{l}\text { 第10天 } \\
\text { 10th day }\end{array}$ \\
\hline$\overline{\mathrm{M} 1}$ & $1.89 \pm 0.18$ & $2.10 \pm 0.19$ & $2.18 \pm 0.22$ & $2.31 \pm 0.20$ & $0.27 \pm 0.05$ & $0.45 \pm 0.08$ & $0.29 \pm 0.06$ & $0.38 \pm 0.07$ \\
\hline M2 & $2.23 \pm 0.18$ & $2.57 \pm 0.16$ & $2.87 \pm 0.22$ & $2.72 \pm 0.20$ & $0.40 \pm 0.05$ & $0.75 \pm 0.09$ & $0.82 \pm 0.09$ & $0.75 \pm 0.09$ \\
\hline M3 & $2.52 \pm 0.19$ & $3.24 \pm 0.23$ & $3.20 \pm 0.18$ & $3.12 \pm 0.23$ & $0.48 \pm 0.05$ & $0.95 \pm 0.11$ & $0.83 \pm 0.11$ & $0.83 \pm 0.11$ \\
\hline M4 & $2.20 \pm 0.18$ & $2.42 \pm 0.18$ & $2.42 \pm 0.24$ & $2.40 \pm 0.17$ & $0.29 \pm 0.03$ & $0.55 \pm 0.06$ & $0.64 \pm 0.08$ & $0.60 \pm 0.08$ \\
\hline M5 & $2.48 \pm 0.16$ & $2.27 \pm 0.18$ & $2.25 \pm 0.16$ & $2.27 \pm 0.15$ & $0.44 \pm 0.07$ & $0.72 \pm 0.09$ & $0.65 \pm 0.07$ & $0.58 \pm 0.06$ \\
\hline M6 & $2.37 \pm 0.15$ & $2.30 \pm 0.15$ & $2.51 \pm 0.16$ & $2.96 \pm 0.26$ & $0.39 \pm 0.05$ & $0.68 \pm 0.09$ & $0.73 \pm 0.10$ & $0.61 \pm 0.08$ \\
\hline M7 & $2.60 \pm 0.18$ & $2.37 \pm 0.17$ & $2.60 \pm 0.19$ & $2.52 \pm 0.17$ & $0.41 \pm 0.05$ & $0.74 \pm 0.13$ & $0.66 \pm 0.07$ & $0.66 \pm 0.08$ \\
\hline M8 & $2.83 \pm 0.37$ & $2.83 \pm 0.27$ & $2.81 \pm 0.34$ & $1.99 \pm 0.17$ & $0.58 \pm 0.15$ & $0.53 \pm 0.09$ & $0.59 \pm 0.18$ & $0.62 \pm 0.17$ \\
\hline M9 & $2.83 \pm 0.29$ & $2.57 \pm 0.33$ & $2.78 \pm 0.41$ & $2.61 \pm 0.47$ & $0.47 \pm 0.16$ & $0.59 \pm 0.14$ & $0.54 \pm 0.07$ & $0.48 \pm 0.07$ \\
\hline M10 & $2.21 \pm 0.29$ & $1.67 \pm 0.25$ & $0.99 \pm 0.13$ & $0.93 \pm 0.13$ & $0.18 \pm 0.03$ & $0.22 \pm 0.06$ & $0.19 \pm 0.02$ & $0.21 \pm 0.06$ \\
\hline M11 & $2.23 \pm 0.26$ & $2.72 \pm 0.42$ & $1.87 \pm 0.24$ & $1.96 \pm 0.27$ & $0.24 \pm 0.05$ & $0.28 \pm 0.04$ & $0.22 \pm 0.03$ & $0.45 \pm 0.16$ \\
\hline M12 & $3.82 \pm 0.40$ & $3.56 \pm 0.38$ & $3.38 \pm 0.45$ & $3.09 \pm 0.31$ & $0.64 \pm 0.11$ & $0.72 \pm 0.08$ & $0.96 \pm 0.20$ & $1.16 \pm 0.25$ \\
\hline
\end{tabular}

表2 黑化的变色沙蚚与非黑化的草原沙蚚各测量部位移植实验前后反射率的配对 $t$ 检验

Table 2 Paired $t$ test analyses on reflectivity for each site in lizards before and after transplantation

\begin{tabular}{|c|c|c|c|c|c|c|}
\hline \multirow{3}{*}{$\begin{array}{l}\text { 检测部位 } \\
\text { Sites }\end{array}$} & \multicolumn{3}{|c|}{ 非黑化的草原沙蜊 “Non-melanistic” P. frontalis } & \multicolumn{3}{|c|}{ 黑化的变色沙蜥 “Melanistic” P. versicolor } \\
\hline & 第1天vs.第8天 & 第1天vs.第9天 & 第1天vs.第10天 & 第1天vs.第8天 & 第1天vs.第9天 & 第1天vs.第10天 \\
\hline & $\begin{array}{l}\text { 1st day vs. 8th day } \\
t_{28}=1.085, P=0.287\end{array}$ & $\begin{array}{l}\text { 1st day vs. 9th day } \\
t_{t_{7}}=1.346, P=0.189\end{array}$ & $\begin{array}{l}1 \text { st day } \\
t_{t_{7}=1.7}\end{array}$ & $\begin{array}{l}\text { 1st day vs. 8th day } \\
t_{28}=1.660, P=0.108\end{array}$ & $\begin{array}{l}\text { 1st day vs. 9th day } \\
t_{28}=1.659, P=0.109\end{array}$ & $\begin{array}{l}\text { 1st day vs. 10th day } \\
t_{27}=1.646, P=0.112\end{array}$ \\
\hline M2 & $t_{28}=1.382, P=0.178$ & $t_{27}=1.724, P=0.096$ & $t_{27}=1.990, P=0.057$ & $t_{28}=3.598, P=0.001^{*}$ & $t_{27}=4.297, P<0.001^{*}$ & $t_{27}=3.099, P=0.005^{*}$ \\
\hline M3 & $376, P=0.025^{*}$ & $t_{27}=3.773, P=0.001^{*}$ & $t_{27}=1.828, P=0.079$ & $t_{27}=4.385, P<0.001^{*}$ & $t_{27}=3.051, P=0.005^{*}$ & $t_{27}=2.995, P=0.006^{*}$ \\
\hline M4 & $t_{28}=0.741, P=0.465$ & $t_{27}=0.530, P=0.600$ & $t_{27}=0.515, P=0.611$ & $t_{28}=3.669, P=0.001^{*}$ & $t_{27}=3.749, P=0.001^{*}$ & $t_{27}=3.438, P=0.002^{*}$ \\
\hline M5 & $t_{28}=-0.644, P=0.525$ & $t_{28}=-0.909, P=0.372$ & $t_{27}=-1.476, P=0.152$ & $t_{28}=2.473, P=0.020^{*}$ & $t_{28}=2.205, P=0.036^{*}$ & $t_{27}=1.611, P=0.119$ \\
\hline M6 & $t_{27}=-0.348, P=0.731$ & $t_{26}=0.467, P=0.644$ & $t_{23}=1.593, P=0.126$ & $t_{27}=-2.686, P=0.012^{*}$ & $t_{26}=-3.366, P=0.002^{*}$ & $t_{23}=2.248, P=0.035^{*}$ \\
\hline M7 & $t_{28}=-1.010, P=0.321$ & $t_{28}=-0.383, P=0.705$ & $t_{27}=-0.759, P=0.455$ & $t_{28}=-2.472, P=0.020^{*}$ & $t_{28}=-2.891, P=0.007^{*}$ & $t_{27}=2.686, P=0.012^{*}$ \\
\hline M8 & $t_{16}=-0.068, P=0.947$ & $t_{16}=-0.111, P=0.913$ & $t_{16}=-1.789, P=0.095$ & $t_{16}=-0.527, P=0.606$ & $t_{16}=-0.396, P=0.698$ & $t_{16}=-0.100, P=0.922$ \\
\hline M9 & $t_{17}=0.059, P=0.954$ & $t_{17}=0.552, P=0.589$ & $t_{17}=0.126, P=0.902$ & $t_{17}=-0.208, P=0.838$ & $t_{17}=0.005, P=0.996$ & $t_{17}=-0.229, P=0.822$ \\
\hline M10 & $t_{16}=-1.477, P=0.162$ & $t_{16}=-4.168, P=0.001^{*}$ & $t_{16}=-4.049, P=0.001^{*}$ & $t_{16}=0.639, P=0.533$ & $t_{16}=0.046, P=0.663$ & $t_{16}=0.564, P=0.582$ \\
\hline M11 & $t_{16}=1.092$, & $t_{16}=-0.899, P=0.384$ & $t_{16}=-0.259, P=0.799$ & $t_{16}=0.349, P=0.733$ & $t_{16}=-0.405, P=0.691$ & $t_{16}=-1.018, P=0.372$ \\
\hline M12 & $t_{16}=-0.261, P=0.798$ & $t_{16}=-0.605, P=0.555$ & $t_{16}=-1.630, P=0.125$ & $t_{16}=1.428, P=0.175$ & $t_{16}=-1.847, P=0.086$ & $t_{16}=2.235, P=0.051$ \\
\hline
\end{tabular}

异; 左后肢(M10)区域第一天的皮肤光反射率与移 植饲养后第 9 九天和第 10 天的皮肤光反射率存在显 著差异; 而其他部位移植饲养前后的光反射率并无 显著差异。

\section{3 讨论}

我们的研究结果表明, 黑化生境下的变色沙蜘 体色显著深于非黑化枯黄色沙丘生境下生存的草 原沙蚚。生境互换围栏移植实验结果表明，饲养1 周后, 黑化种群背部6个检测部位(M2-M7)的光反 射率统计值显著升高, 其他部位均无显著变化; 而 非黑化种群只有背部右上方和左后肢 2 个部位(M3
和M10)的皮肤光反射率统计值发生显著变化, 其 他部位反射率并无显著变化。虽然一些部位有统计 差异, 但种群内样本体色变异程度非常轻微, 甚至 肉眼难以识别是否发生体色变化。

动物体色变异的机制主要有生理体色变异和 形态体色变异两种(Devi \& Adnan, 2009)。对基底颜 色的匹配可以降低动物在外出活动时被捕食者及 猎物发现的可能性(Merilaita et al, 2001; Stuart-Fox et al, 2003; Vignieri et al, 2010), 而短期内快速匹配 基底颜色的能力主要依赖于色素颗粒的快速扩散 与聚集(即生理体色变化)(Nery \& Castrucci, 1997)。 研究发现部分鱼类以及一些两栖爬行类动物中存 
在着生理上的快速体色变化, 并能在短时间内匹配 基底颜色(Mäthger et al, 2003; Boback \& Siefferman, 2010; Choi \& Jang, 2014)。我们的研究发现, 两种沙 蚚群体自然体色变化存在显著差异 $(P<0.05)$, 相比 于上述快速的生理体色变化, 互换环境饲养1周至 10 天后沙蜥样本体色与饲养时所处的基底颜色依 然存在很大差异, 并且肉眼难以区分是否发生了显 著的体色变化。这说明, 对基底生境颜色的短期适 应变化并不能解释不同基底色生境中两种沙蚚所 具有的显著的自然体色差异。

此外, 有研究发现, 在人为制造的具有不同颜 色深度的基底环境中, “浅色”动物个体的体色变化 程度通常要比黑化个体大(Norris, 1965; Schlichting \& Pigliucci, 1999; Rosenblum, 2004), 这是因为相比 于 “浅色”个体，黑化个体由于增加了皮肤黑色素的 产量来匹配起初的黑化基底环境, 在短期非黑化环 境刺激下聚集和扩散黑色素颗粒的能力相对更弱。 然而, 我们对两种沙蚚生境互换实验结果却发现, 黑化的变色沙蚚种群样本在短期内的体色显著变 化部位比非黑化草原沙蜥种群多, 表明变色沙蜊可 能具有比草原沙蚚更强的体色变异能力。此外, 两 种沙蝰种群显著的自然体色变化并不是色素颗粒 的快速扩散或聚集而形成, 其色素积累或体色变化 在个体发育过程中可能是一个渐进的过程。

本文只比较了成体样本, 未对幼体与亚成体进 行比较, 但这种体色变化显然是不同群体对不同基 底环境的长期适应而形成的稳定的变化。我们的研 究还发现西藏沙蚚(P. theobaldi)低海拔种群没有腹 部黑斑, 而海拔 4,200 m以上的种群出现腹部黑斑, 且腹部黑斑面积随海拔上升显著变大, 并认为这是 对不同温度环境的一种热适应(Jin \& Liao, 2015)。 而本研究选择的两种沙蚚群体海拔分布相近, 但基 底颜色显著不同, 各沙蜊群体体色保持与基底颜色 相近，有利于其反捕食。

动物体皮肤黑化的程度跟黑色素细胞的活性 相关(Alibardi, 2013), 一些动物表皮黑色素细胞层 可能需要数天或数月时间将黑素体逐渐转移到邻 近的角质细胞层从而使皮肤黑化(即形态体色变 异)(Cooper \& Greenberg, 1992)。少数研究表明, 与 黑色素合成通路密切相关的基因遗传变异可能在 爬行动物体色变异中扮演重要角色(Rosenblum et $\mathrm{al}, 2004,2010)$ 。本研究表明, 不同基底色生境中生
存的2种沙蚚体色的显著差异并不是对基底生境颜 色的短期适应改变, 而更倾向于是受遗传与进化因 素影响的形态体色变异, 两物种体色表型分化明 显, 其差异体色表型已在各自然种群中稳定遗传, 但潜在的影响机制还需要日后分子生物学与功能 实验进一步揭示。

致谢: 感谢中国计量大学曾炜炜同学的实验协助与 部分数据分析工作。

\section{参考文献}

Alibardi L (2013) Observations on the ultrastructure and distribution of chromatophores in the skin of chelonians. Acta Zoologica, 94, 222-232.

Barlett PN, Gates DM (1966) The energy budget of a lizard on a tree trunk. Ecology, 48, 315-322.

Bennett ATD, Cuthill IC (1994) Ultraviolet vision in birds: what is its function? Vision Research, 34, 1471-1478.

Boback SM, Siefferman LM (2010) Variation in color and color change in island and mainland boas (Boa constrictor). Journal of Herpetology, 44, 506-515.

Cai B (2014) Rapid color variation in reptile animals. Bull- etin of Biology, 49(12), 4-6. (in Chinese) [蔡波 (2014) 爬行动 物体色的快速变化. 生物学通报, 49(12), 4-6.]

Chen Q, Han ZX, Song ZM (1993) A study on the reproduction of lizard Phrynocephalus versicolor. Journal of Lanzhou University, 29, 199-203. (in Chinese with English abstract) [陈强, 韩昭雪, 宋志明 (1993) 变色沙蚚繁殖的研究. 兰 州大学学报, 29, 199-203.]

Choi N, Jang Y (2014) Background matching by means of dorsal color change in treefrog populations (Hyla japonica). Journal of Experimental Zoology Part A Ecological Genetics \& Physiology, 321, 108-118.

Cooper WE, Greenberg N (1992) Reptilian coloration and behavior. In: Biology of the Reptilia, Vol. 18. Physiology E: Hormones, Brain and Behavior (eds Gans C, Crews D), pp. 298-422. University of Chicago Press, Chicago.

Cott HB (1940) Adaptive Coloration in Animals. Methuen and Co. Ltd., London.

Devi SF, Adnan M (2009) Camouflage, communication and thermoregulation: lessons from colour changing organisms. Philosophical Transactions of the Royal Society B: Biological Sciences, 364, 463-470.

Geen MRS, Johnston GR (2014) Coloration affects heating and cooling in three color morphs of the Australian bluetongue lizard, Tiliqua scincoides. Journal of Thermal Biology, 43, 54-60.

Guo L, Zhao CG (2001) Study on the reproductive strategy of lizard Phrynocephalus frontalis. Acta Scientiarum Naturalium Universitatis Neimongol (Natural Science Edition), 32, 214-275. (in Chinese with English abstract) [ 郭砺, 赵辰光 (2001) 草原沙蜥 (Phrynocephalus 
frontalis)生殖策略的研究. 内蒙古大学学报(自然科学版), 32, 214-275.]

Hanlon R (2007) Cephalopod dynamic camouflage. Current Biology, 17, 400-404.

Hemmi JM, Marshall J, Pix W, Vorobyev M, Zeil J (2006) The variable colours of the fiddler crab Uca vomeris and their relation to background and predation. Journal of Experimental Biology, 209, 4140-4153.

Jin YT, Liao PH (2015) An elevational trend of body size variation in a cold-climate agamid lizard, Phrynocephalus theobaldi. Current Zoology, 61, 444-453.

Mäthger LM, Land MF, Siebeck UE, Marshall NJ (2003) Rapid colour changes in multilayer reflecting stripes in the paradise whiptail, Pentapodus paradiseus. Journal of Experimental Biology, 206, 3607-3613.

Merilaita S, Lyytinen A, Mappes J (2001) Selection for cryptic coloration in a visually heterogeneous habitat. Proceedings of the Royal Society of London B: Biological Sciences, 268, 1925-1929.

Moretz JA, Morris MR (2003) Evolutionarily labile responses to a signal of aggressive intent. Proceedings of the Royal Society B: Biological Sciences, 270, 2271-2277.

Nery LEM, Castrucci AMDL (1997) Pigment cell signalling for physiological color change. Comparative Biochemistry \& Physiology Part A Physiology, 118, 1135-1144.

Norris KS (1965) Color adaptation in desert reptiles and its thermal relationships. In: Lizard Ecology: A Symposium (ed. Milstead WW), pp. 162-226. University of Missouri Press, Columbia, Missouri.

Porter WP, Gates DM (1969) Thermodynamic equilibria of animals with environment. Ecological Monographs, 39, 227-244.

Qu YF, Gao JF, Mao LX, Ji X (2011) Sexual dimorphism and female reproduction in two sympatric toad-headed lizards, Phrynocephalus frontalis and P. versicolor (Agamidae). Animal Biology, 61, 139-151.

Quan RZ, Chen DF, Zhang JF (2006) Studies on the hunger-resistance and the feeding habit of Phrynocephalus versicolor. Journal of Shihezi University (Natural Science), 24, 436-438. (in Chinese with English abstract) [全仁哲, 陈道富, 张继锋 (2006) 变色沙蚚 (Phryno- cephalus versicolor)的耐饥能力与食性研究. 石河子大学学报(自 然科学版), 24, 436-438.]

Rosenblum EB, Hoekstra HE, Nachman MW (2004) Adaptive reptile color variation and the evolution of the Mc1r gene. Evolution, 58, 1794-1808.

Rosenblum EB, Römpler H, Schöneberg T, Hoekstrac HE (2010) Molecular and functional basis of phenotypic convergence in white lizards at white sands. Proceedings of the National Academy of Sciences, USA, 107, 2113- 2117.

Schlichting CD, Pigliucci M (1999) Phenotypic evolution: a reaction norm perspective. American Journal of Physical Anthropology, 109, 144-146.

Stegen JC, Gienger CM, Sun LX (2004) The control of color change in the Pacific tree frog, Hyla regilla. Canadian Journal of Zoology, 82, 889-896.

Stuart-Fox DM, Moussalli A, Marshall NJ, Owens IPF (2003) Conspicuous males suffer higher predation risk: visual modelling and experimental evidence from lizards. Animal Behaviour, 66, 541-550.

Stuart-Fox D, Moussalli A, Whiting MJ (2008) Predatorspecific camouflage in chameleons. Biology Letters, 4, 326-329.

Thurman CL (1988) Rhythmic physiological color change in crustacea: a review. Comparative Biochemistry \& Physiology Part C. Comparative Pharmacology, 91, 171-185.

Vignieri SN, Larson JG, Hoekstra HE (2010) The selective advantage of crypsis in mice. Evolution, 64, 2153-2158.

Vroonen J, Vervust B, Fulgione D, Maselli V, Damme RV (2012) Physiological colour change in the Moorish gecko, Tarentola mauritanica (Squamata: Gekkonidae): effects of background, light, and temperature. Biological Journal of the Linnean Society, 107, 182-191.

Wang YZ, Fu JZ (2004) Cladogenesis and vicariance patterns in the toad-headed lizard Phrynocephalus versicolor species complex. Copeia, 2004, 199-206.

Woolley P (1957) Colour change in a chelonian. Nature, 179, 1255-1256.

Yang CC, Cai Y, Liang W (2011) Analysis of the correlation between plumage color and male quality in yellow- throated buntings. Sichuan Journal of Zoology, 30(1), 1-5. (in Chinese with English abstract) [杨灿朝, 蔡燕, 梁伟 (2011) 黄喉坐的羽色与雄鸟质量相关性分析. 四川动物, 30(1), 1-5.]

Yang CC, Liang W (2013) Using spectra and visual modeling to study animal coloration. Zoological Research, 34, 564-573. (in Chinese with English abstract) [杨灿朝, 梁伟 (2013) 通过光谱与视觉模型研究动物体色. 动物学研究, 34, 564-573.]

Zhao EM, Jiang YM, Huang QY, Hu SQ, Fei L, Ye CY (1998) Latin-Chinese-English Names of Amphibians and Reptiles. Science Press, Beijing. (in Chinese) [赵尔宓, 江跃明, 黄 庆云, 胡淑琴, 费梁, 叶昌媛 (1998) 拉汉英两栖爬行动 物名称. 科学出版社, 北京.]

Zhao EM, Zhao KT, Zhou KY (1999) Fauna Sinica, Reptilia Vol. 2, Squamata, Lacertilia. Science Press, Beijing. (in Chinese) [赵尔宓, 赵肯堂, 周开亚 (1999) 中国动物志, 爬行纲第二卷, 有鳞目, 蜥蜴亚目. 科学出版社, 北京.]

Zhao X, Bi JH, Liu R, He ZC, Chen SY (2013) The feeding habits of toad-headed lizard (Phrynocephalus frontalis) in autumn. Chinese Journal of Zoology, 48, 321-330. (in Chinese with English abstract) [赵雪, 毕俊怀, 刘睿, 何志 超, 陈绍勇 (2013) 草原沙蚚秋季食性分析. 动物学杂 志, 48, 321-330.]

(责任编委：傅金钟 责任编辑：时意专) 